\title{
Nuevas estrategias metodológicas para incentivar el estudio y el trabajo cooperativo en el contexto universitario
}

Francisca Sempere Ferre

Departamento de Estadística e Investigación Operativa, Escuela Técnica Superior de Ingeniería del Diseño, Universidad Politécnica de Valencia, Valencia 46022, España. frasemfe@upvnet.upv.es

\begin{abstract}
Many students currently entering at the university, lack of study habits. In order to encourage these, increase the motivation and improve the learning of the subject, a new methodological strategy was proposed. The research was carried out in a group of 40 students. Groups of four people were established randomly. Consecutively, each group chose a question at random from 70 questions of different types. For each correct answer, the team received a piece of a puzzle to configure a figure. The winning team was assigned 0.1 from the final score of the subject. The experiment was carried out three times during the quarter with different questions. $95 \%$ of students evaluated the activity positively, showing a high degree of motivation and satisfaction in teamwork.
\end{abstract}

Keywords: study habits, learning, motivation, cooperative work, methodology, human physiology.

\section{Resumen}

Muchos estudiantes que ingresan actualmente en la universidad, carecen de hábitos de estudio. Con el objetivo de incentivar estos, aumentar la motivación y mejorar el aprendizaje de la asignatura se planteó una nueva estrategia metodológica. La investigación se realizó en un grupo de 40 alumnos Se establecieron grupos de cuatro personas de forma aleatoria. Consecutivamente cada grupo eligió una pregunta al azar entre 70 cuestiones formuladas de distinta tipología. Por cada respuesta acertada, el equipo recibió una pieza de un puzle para configurar una figura. Al equipo ganador se le asignó un 0,1 de la nota final de la asignatura. El experimento se realizó tres veces durante el cuatrimestre con diferentes cuestiones. El $95 \%$ de los estudiantes valoraron positivamente la actividad, mostrando un alto grado de motivación y satisfacción en el trabajo en equipo.

Palabras clave: hábitos de estudio, aprendizaje, motivación, trabajo cooperativo, metodología, fisiología humana.

\section{Introducción}

Los hábitos de estudio son los métodos y estrategias que acostumbra a usar el estudiante para asimilar contenidos, su aptitud para evitar distracciones, su atención al material específico y los esfuerzos que realiza a lo largo de todo el proceso (Cartagena, 2008). 
Muchos estudiantes que ingresan actualmente en la universidad carecen de estos hábitos (Acevedo et al., 2015; Hernández Herrera et al., 2012). Evidentemente en este escenario, la motivación juega un papel fundamental ya que los alumnos motivados aprenden con mayor rapidez y más eficazmente, que los que no lo están.

En este sentido, es necesario que el docente del siglo XXI desarrolle estrategias para conseguir mejorar el rendimiento académico del alumno. Aunque la sociedad actual nos ofrece múltiples herramientas enfocadas a conseguir este objetivo, a veces con pocos medios, se pueden alcanzar los mismos resultados.

\section{Objetivos}

El objetivo general que se planteó al implantar esta estrategia metodológica fue:

- Incentivar el aprendizaje de la asignatura.

Objetivos especifícos:

- Fomentar el hábito de estudio entre los estudiantes universitarios.

- Motivar al alumno a través de la competitividad.

- Estimular el trabajo cooperativo.

\section{Desarrollo de la innovación}

\subsection{Asignatura}

El experimento se realizó en la asignatura de Fisiología Humana I del Grado de Nutrición Humana y Dietética (Universidad Católica de Valencia San Vicente Mártir) durante el curso académico 2018-2019 con una muestra poblacional de 40 alumnos.

La asignatura pretende dar a conocer al alumno el funcionamiento del organismo humano en su conjunto, entendido como resultado de la correcta interacción de los diversos procesos celulares y moleculares que lo integran.

\subsection{Equipos}

La configuración de los equipos fue realizada por el profesor de forma aleatoria. Cada equipo estuvo constituido por 4 alumnos. A cada grupo se le asignó un número.

\subsection{Preguntas}

El profesor formuló 70 preguntas vinculadas con los diferentes contenidos de la asignatura y a cada cuestión le fue asignada un número de forma correlativa.

La tipología de las preguntas fue de diferentes tipos: verdadero o falso, preguntas cortas cerradas y preguntas de opción múltiple.

En el turno correspondiente a cada grupo, la elección de la pregunta se realizó por los alumnos al azar eligiendo un número del 1 al 70 y sin conocer el contenido de la misma. Una vez formulada la pregunta, para no repetirse esta fue eliminada del documento. 


\subsection{Comodines}

Con el objetivo de incentivar la competitividad, según los criterios del profesor, a algunas preguntas se les asignó un comodín:

- Comodín amarillo: pasar la pregunta a otro grupo (elegido por los integrantes del equipo).

- Comodín azul: no contestar a la pregunta formulada y elegir otro número.

- Comodín rosa: Se contesta a la pregunta mirando los apuntes.

- Comodín verde: El grupo se lleva el punto sin contestar la pregunta y rebota está a otro grupo (elegido por los integrantes del equipo).

La materialización de estos se realizó utilizando cartulinas de distintos colores.

El grupo podía utilizar el comodín en su turno de respuesta o guardarlo para usarlo posteriormente.

\subsection{Tiempo de respuesta}

Una vez formulada la pregunta, el grupo dispuso de 1 minuto para consensuar una respuesta entre los miembros del equipo. Se utilizó un temporizador online que se proyectó en la pizarra (Figura 1).

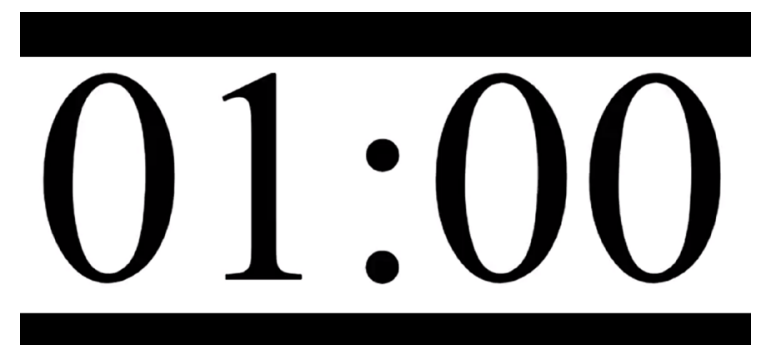

Fig. 1. Temporizador utilizado en el proyecto.

\subsection{Puntuación}

Cada pregunta contestada correctamente fue valorada con una pieza de un puzle. Una vez reunidas todas las piezas del puzle el equipo tuvo que configurarlo.

Las 10 piezas del puzle conformaban una imagen vinculada con los contenidos de la asignatura.

Al equipo ganador, que consiguió montar la figura primero, se les asignó un 0,1 sobre la nota final de la asignatura.

\subsection{Realización del experimento}

Las cuestiones se formularon siguiendo el orden numérico de los grupos. Una vez formulada la pregunta y transcurrido el tiempo (1 minuto), el profesor eligió al azar un alumno para su respuesta, no siendo vinculante esta elección para posteriores turnos.

La nueva estrategia metodológica se realizó tres veces durante el cuatrimestre, incorporando diferentes cuestiones según los contenidos de la asignatura impartidos hasta el momento. 


\subsection{Análisis de los resultados}

Para comprobar que se habían alcanzado los objetivos propuestos, al final de la sesión se pasó una encuesta diseñada con Google Forms.

\section{Resultados}

\subsection{Valoración metodológica}

Al preguntarles a los alumnos si la estrategia metodológica seguida puede mejorar los hábitos de estudio e incentivar el aprendizaje de la asignatura, el 95\% de los encuestados contestó que sí (Figura 2).

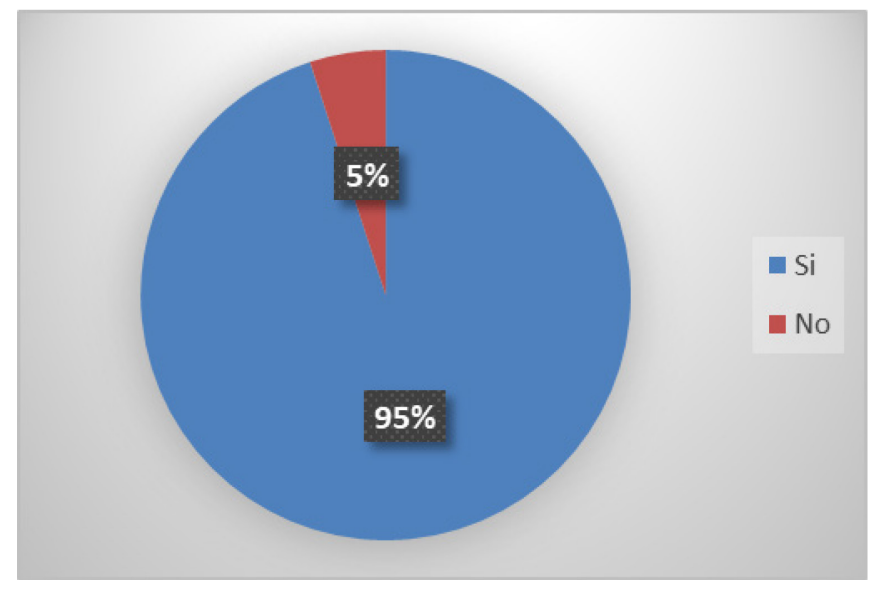

Fig. 2. Valoración de la metodología como herramienta de aprendizaje.

\subsection{Trabajo cooperativo}

La elección aleatoria dentro de los miembros del grupo para contestar la pregunta formulada, consiguió que todos los alumnos estuvieran centrados en la sesión.

Para evaluar el grado de satisfacción del trabajo en equipo se establecieron 5 grados siguiendo la escala Likert: no satisfecho, poco satisfecho, moderadamente satisfecho, muy satisfecho, extremadamente satisfecho.

El 75\% de los alumnos se mostraron extremadamente satisfechos, mientras que el $15 \%$ muy satisfechos y un $5 \%$ satisfechos (Figura 3). 


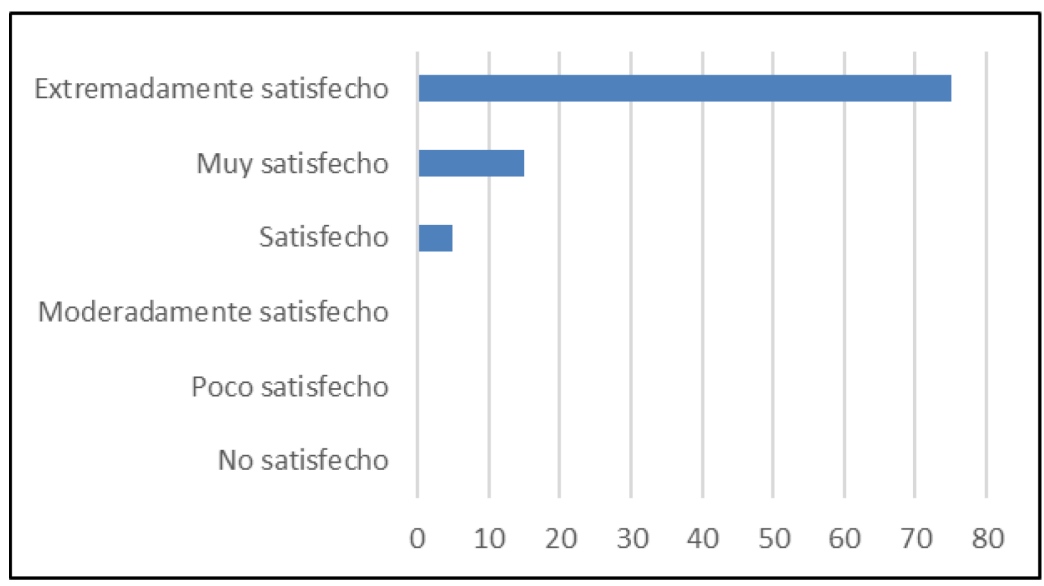

Fig. 3. Valoración del trabajo en equipo.

\subsection{Grado de motivación}

El grado motivación se midió siguiendo una escala Likert de 1 a 5 (muy bajo, bajo, medio y alto y muy alto).

La mayoría de los alumnos respondió que el grado de motivación al realizar la actividad fue muy alto $(97,5 \%)$ y tan solo para un alumno el grado de motivación fue alto (Figura 4).

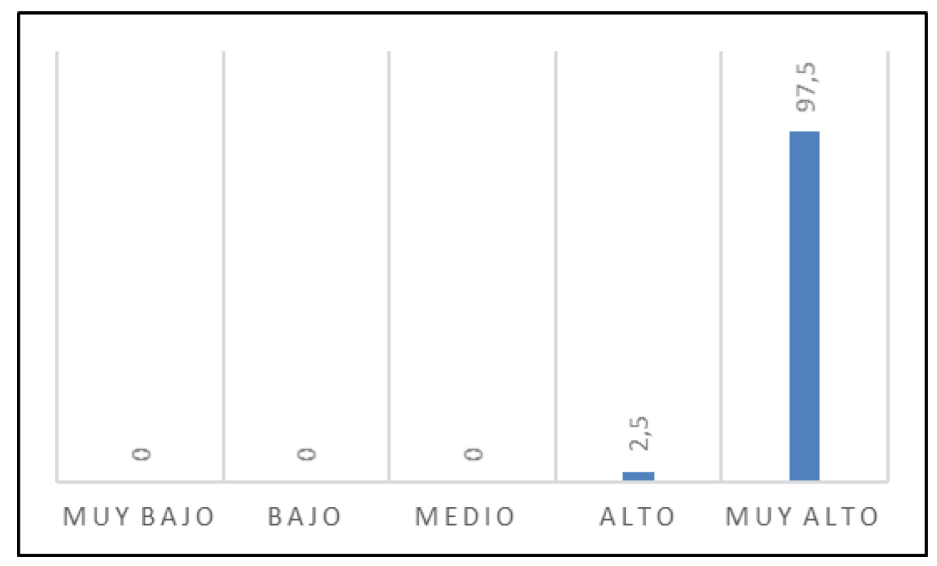

Fig. 4. Valoración del grado de motivación en la estrategia metodología utilizada.

\section{Conclusiones}

Con este estudio se demuestra que la implementación de esta estrategia metodológica puede mejorar los hábitos de estudio del alumnado, así como la motivación y el trabajo cooperativo, repercutiendo de manera satisfactoria sobre el aprendizaje de la asignatura.

Hacen falta más investigaciones al respecto, pero según la experimentación realizada, podría ser una buena metodología para utilizar en el ámbito universitario. 


\section{Referencias}

ACEVEDO, D. TORRES, J. D. y TIRADO, D. F. (2015). “Análisis de los Hábitos de Estudio y Motivación para el Aprendizaje a Distancia en Alumnos de Ingeniería de Sistemas de la Universidad de Cartagena (Colombia)". Formación universitaria, vol 8(5), pp. 59-66.

$<$ https://dx.doi.org/10.4067/S0718-50062015000500007> [Consulta: 28 de julio de 2020].

CARTAGENA, M. (2008). "Relación entre la autoeficacia, el rendimiento escolar y los hábitos de estudio de secundaria" en Revista Iberoamericana sobre Calidad, Eficacia y cambio en Educación, vol. 6 (3).

HERNÁNDEZ HERRERA, C. A. RODRÍGUEZ PEREGO, N. y VARGAS GARZA, A. E. (2012). "Los hábitos de estudio y motivación para el aprendizaje de los alumnos en tres carreras de ingeniería en un tecnológico federal de la ciudad de México" en Revista de la educación superior, vol 41(163), pp. 67-87.

$<$ http://www.scielo.org.mx/scielo.php?script=sci_arttext\&pid=S018527602012000300003\&lng=es\&tlng=es $>$ [Consulta: 28 de julio de 2020]. 